\title{
A Preliminary Model of Centering in Dialog*
}

\author{
D. Byron and A. Stent \\ University of Rochester Computer Science Department \\ Rochester NY 14627, U.S.A. \\ dbyron/stent@cs.rochester.edu
}

\begin{abstract}
The centering framework explains local coherence by relating local focus and the form of referring expressions. It has proven useful in monolog, but its utility for multiparty discourse has not been shown, and a variety of issues must be tackled to adapt the model for dialog. This paper reports our application of three naive models of centering theory for dialog. These results will be used as baselines for evaluating future models. ${ }^{1}$
\end{abstract}

\section{Introduction}

The centering framework (Grosz et al., 1995) is one of the most influential computational linguistics theories relating local focus to the form chosen for referring expressions. A number of studies have developed refinements and extensions of the theory (eg. Brennan et al., 1987; Kameyama, 1986; Strube and Hahn, 1996; Walker et al., 1998), but few have attempted to extend the model to multi-party discourse (cf. Brennan, 1998; Walker, 1998).

For dialog systems, the benefits of using centering theory include improved reference resolution and generation of more coherent referring expressions. However, it is not at all clear how to adapt the theory for multi-party discourse. This paper examines some of the issues involved in adapting the theory, then describes the results of applying three alternative models to a corpus of 2-person dialogs. We chose very naive approximations to the original theory as a starting point. These results will be a baseline for evaluating more sophisticated models in the future.

\section{The Centering model}

The centering framework (Grosz et al., 1995) makes three main claims: 1) given an utterance $U_{n}$, the

\footnotetext{
- The authors would like to thank James Allen, Marilyn Walker, and the anonymous reviewers for many helpful comments on a preliminary draft of the paper. This material is based on work supported by NSF grant IRI-96-23665, ONR grant N00014-95-1-1088 and Columbia University grant OPG:1307.

${ }^{1}$ A more detailed report of this study is available as URCS TR \#687 (Byron and Stent, 1998)
}

model predicts which discourse entity will be the focus of $U_{n+1} ; 2$ ) when local focus is maintained between utterances, the model predicts that it will be expressed with a pronoun; and 3) when a pronoun is encountered, the model provides a preference ordering on possible antecedents from the prior utterance.

These data structures are created for each $U_{n}:{ }^{2}$

1. A partially-ordered list of forward-looking centers $C f_{n}$ that includes all discourse entities in utterance $n$. Its first element is the 'preferred center', $C p_{n}$.

2. A backward-looking center $C b_{n}$, the highest ranked element of $C f_{n-1}$ that is in $C f_{n}$.

The framework defines a preference ordering on techniques for effecting a topic change, ranked according to the inference load each places on the addressee. The transitions are called 'shift', 'retain' and 'continue' and differ based on whether $C b_{n}=C b_{n+1}$ and whether $C b_{n}=C p_{n}$.

At the heart of the theory are two centering rules: Rule 1: If any member of $C f_{n}$ is realized by a pronoun in $C f_{n+1}, C b_{n+1}$ must be a pronoun.

Rule 2: Sequences of continues are preferred over sequences of retains, and sequences of retains are preferred over sequences of shifts.

\section{Centering and multi-party discourse}

A variety of issues must be addressed to adapt centering to two-party dialog. They include:

1. Utterance boundaries are difficult to pin down in spoken dialog, and their determination affects the $C f$ lists. Just how the speaker turns are broken into utterances has a huge impact on the success of the model (Brennan, 1998).

2. Should the dialog participants, referred to via first- and second-person pronouns (1/2PPs), be considered 'discourse entities' and included in $C f$ ?

\footnotetext{
${ }^{2}$ We provide only the briefest sketch of the centering framework. Readers unfamiliar with the model are referred to (Grosz et al., 1995) for more details.
} 
3. Which utterance should be considered 'previous' for locating $C f_{n-1}$ : the same speaker's previous utterance or the immediately preceding utterance, regardless of its speaker?

4. What should be done with abandoned or partial utterances and those with no discourse entities.

\section{Experimental method}

Our data is from four randomly chosen dialogs in the CALLHOME-English corpus ${ }^{3}$ (LDC, 1997). Table 1 describes the three models we created to address the issues described in Section 3.

\begin{tabular}{lcc}
\hline & $\begin{array}{c}C f \text { elements } \\
\text { from } 1 / 2 \mathrm{PPS}\end{array}$ & $\begin{array}{c}\text { Use both speakers' } \\
\text { previous utt to find } \mathrm{Cb}\end{array}$ \\
\hline Model 1 & Yes & No \\
Model 2 & No & Yes \\
Model 3 & No & No \\
\hline
\end{tabular}

Table 1: The Centering Models

Issue 1: Utterance boundaries We honored utterance boundaries as transcribed ${ }^{4}$, even if an utterance was a fragment properly belonging at the end of the one preceding. For instance, the following two utterances seem as though they should be just one:

Example I[dialog 4571]
A ... and she called me one day when
A there was nobody in the house but her...

For compound sentences, we broke each nonsubordinate clause into a new utterance. The utterance break added in Example 2 is indicated by /:

Example 2 [dialog 4248]
A $\quad$ It does make a difference / like I always
thought formula smells kind of disgusting.

Issue 2: Selection of items for Cf Two crucial factors in the original model are left to the algorithm implementer: the selection of items for $C f$ and their rank order. Both are active areas of research. In our models, all elements of $C f$ are created from nouns in the utterance. We do not include entities referred to by complex nominal constituents such as infinitives. Associations (eg. part/subpart) and ellipsed items are not allowed in determining elements of $C f$. We adopted a commonly used $C f$ ordering: Subj $>\mathrm{DO}>\mathrm{IO}>$ Other. Linear sentence position is used to order multiple 'other' constituents. Whether discourse participants should be considered discourse entities is very perplexing

\footnotetext{
${ }^{3}$ The dialog transcripts consisted of 614 utterances, $30 \mathrm{~min}$ utes of speech. After annotation (see issue 1 in section 4), there were 664 non-empty utterances.

${ }^{4}$ CALLHOME transcribers separated utterances at a speaker change or a long pause, or if the semantics or syntax of the language indicated the end of an utterance.
}

from a centering viewpoint (Byron and Stent, 1998). One of our models includes entities referred to by 1/2PPs in $C f$ and two do not.

Issues 3/4: Previous utterance Empty utterances (containing no discourse entities) are skipped in determining $C f_{n-1}$. Empty utterances include acknowledgements and utterances like "hard to leave behind" with no explicitly mentioned objects. The dialogs were annotated for discourse structure, so $U_{n-1}$ is the previous utterance in the discourse segment, not necessarily linear order. ${ }^{5}$ In model2, the highest ranked element of $C f$ from either the current speaker's prior utterance or the other speaker's previous utterance is $C b^{6}$; models $1 \& 3$ consider only the immediately preceding utterance.

We also annotated the 'real' topic of each utterance, selected according to the annotator's intuition of what the utterance is 'about'. It must be explicitly referred to in the utterance and can be an entity referred to using a $1 / 2 \mathrm{PP}$.

After the three models were defined, one dialog was used to train the annotators (the authors) ${ }^{7}$, then the other three were independently annotated according to the rules outlined above. The annotators compared their results and agreed upon a reconciled version of the data, which was used to produce the results reported in Section 5. Annotator accuracy as measured against the reconciled data over all categories ranged from $80 \%$ to $89 \%$. Accuracy was calculated by counting the number of utterances that differed from the reconciled data (including different ordering of $C f$ ), divided by total utterances. ${ }^{8}$

\section{Results and analysis}

Table 2 summarizes our findings. Only 10 of 664 utterances violate Centering Rule 1 , so centering theory's assumptions linking local focus to pronouns appear to hold in dialog. It is interesting to note that Model 1, which includes dialog participants as discourse entities, consistently performed best in the categories used for this evaluation. ${ }^{9}$

\footnotetext{
${ }^{5}$ The authors performed segmentation together; the purpose of this study is to examine extensions of centering theory, not discourse segmentation.

${ }^{6}$ In case of conflict, recency takes precedence.

${ }^{7}$ Annotators must not confer during annotation, so a training dialog is used to clarify unclear annotation instructions. In this case, the annotators examined it to agree on which syntactic constituents would contribute $C f$ elements and the criteria for breaking turns into utterances.

${ }^{8}$ More standard reliability measures could not be used since there are no "tags" in this annotation scheme, and within some categories there may be an ordered list of items.

${ }^{9}$ But see (Byron and Stent, 1998).
} 


\begin{tabular}{|c|c|c|c|c|c|c|c|c|c|c|c|c|}
\hline & \multicolumn{3}{|c|}{ empty $C b$} & \multicolumn{3}{|c|}{$C b=$ topic } & \multicolumn{3}{|c|}{ chan transitions } & \multicolumn{3}{|c|}{ expensive trans. } \\
\hline & M1 & M2 & M3 & M1 & M2 & M3 & & M2 & M3 & & M2 & M3 \\
\hline $\begin{array}{l}\text { Dialog 1: } 227 \text { utts } \\
\text { Dialog 2: } 229 \text { utts } \\
\text { Dial 3: } 208 \text { uts }\end{array}$ & $\begin{array}{l}110 \\
105 \\
103\end{array}$ & $\begin{array}{l}156 \\
174 \\
137\end{array}$ & $\begin{array}{l}169 \\
176 \\
139\end{array}$ & $\begin{array}{l}71 \\
87 \\
77\end{array}$ & $\begin{array}{l}49 \\
41 \\
54\end{array}$ & $\begin{array}{l}47 \\
38 \\
54\end{array}$ & $\begin{array}{l}94 \\
93 \\
84\end{array}$ & $\begin{array}{l}48 \\
37 \\
58\end{array}$ & $\begin{array}{l}47 \\
37 \\
58\end{array}$ & $\begin{array}{l}133 \\
136 \\
114\end{array}$ & $\begin{array}{l}744 \\
149 \\
123\end{array}$ & $\begin{array}{l}145 \\
149 \\
143\end{array}$ \\
\hline$\overline{\Sigma \text { for all dialogs }}$ & 318 & 4467 & $\overline{484}$ & 235 & 144 & 139 & 271 & 143 & $\overline{142}$ & 383 & 416 & 417 \\
\hline & $48 \%$ & & $73 \%$ & & $\begin{array}{l}1 \text { utts } \\
22 \%\end{array}$ & $21 \%$ & $41 \%$ & $26 \%$ & $25 \%$ & & $74 \%$ & $75 \%$ \\
\hline
\end{tabular}

Table 2: Comparison of three alternative centering models for dialog

\subsection{Empty Cb's}

Each of our models leaves at least $52 \%$ of nonempty utterances with no prediction of the $C b$ $\left(C f_{n-1}\right.$ and $C f_{n}$ are disjoint). ${ }^{10}$ Some empty $C b$ 's result from abrupt topic shifts, while others occur when the speakers make topically related, but $C f$-disjoint, contributions, such as the last line in:

Example 3 [dialog 4861]
A I just want to figure out what I'm going to do with my life. I feel like I'm never going to figure it out.

B Lizzy, you might not.

B I haven't figured out mine yet.

In many cases, a $C b$ would exist if we modified the models to include associated and ellipsed entities in $C f$. For instance, in Example 4, the ellipsed location in A's utterance should be the $C b$ :

\section{Example 4 d dialog 4248]}

B ... I've been there wait, yes three times I think

A Well this is our second time

\subsection{Cb Matches the 'real' topic}

For utterances where a $C b$ can be selected, it matches the 'real' topic only $21 \%$ to $35 \%$ of the time. By this measure, our models are poor predictors of local focus. For instance, in Example 5 , the 'real' topic of the first utterance is Jackson, but according to Model 1 the set of entities referred to by "we" is the $C b$ of both utterances.

\section{Example 5 [dialog 4248]}

A And like we went into Jackson, the town and / we were like - AAAHHH! let me out of here

The annotators' intuitions regarding the 'real' topic often conflicted. It would be interesting to annotate actor and discourse focus separately, then see which one the $\mathrm{Cb}$ most closely matches.

\subsection{Cheap versus expensive transitions}

Strube and Hahn (1996) propose a method of evaluating a model against centering rule 2 , measuring the 'cost' of the listener's inference load. A cheap transition has $C b_{n}=C p_{n-1}$, otherwise it is expensive. Models with a large percent of cheap transi-

\footnotetext{
${ }^{10} 57 \%$ of $C b^{\prime}$ 's in Modell are entities referred to via $1 / 2 \mathrm{PPs}$.
}

tions better reflect human notions of coherence. All three of our models produced a very low percent of cheap transitions in this experiment, especially when compared to Strube and Hahn's result of $80 \%$.

\section{Conclusions and Future work}

We conclude that centering behavior in dialog is consistent with that found in monolog. However, the utility of our preliminary models is questionable. By revising our Model 1, we believe a useful model of centering in dialog can be built.

This study indicates many promising directions for future research. Some we intend to pursue are:

- Evaluate the models using other criteria, e.g. improved pronoun resolution.

- Experiment with alternate $C f$ orderings and improve the semantic theory to include entities referred to by personal pronouns, associations and ellipsed entities in $C f$.

- Modify utterance boundaries to re-attach interrupted utterances or use Kameyama's proposal for 'center update units' (1998).

\section{References}

Brennan, Friedman, and Pollard. 1987. A centering approach to pronouns. In Proceedings of $A C L$ ' 87 .

Susan E. Brennan. 1998. Centering as a psychological resource for achieving joint reference in spontaneous discourse. In (Walker et al., 1998).

D. Byron and A. Stent. 1998. A preliminary model of centering in dialog. Technical Report 687, University of Rochester CS Department. http: //www. cs.rochester. edu/trs.

Grosz, Joshi, and Weinstein. 1995. Centering: A framework for modeling the local coherence of discourse. Computational Linguistics, 21(2).

Megumi Kameyama. 1986. A property-sharing contraint in centering. In Proceedings of $A C L$ ' 86 .

Megumi Kameyama. 1998. Intrasentential centering: A case study. In (Walker et al., 1998).

1997. CALLHOME American English Speech. Linguistics Data Consortium.

Michael Strube and Udo Hahn. 1996. Functional centering. In Proceedings of $A C L$ ' 96.

Walker, Joshi, and Prince, editors. 1998. Centering Theory in Discourse. Clarendon Press, Oxford.

Marilyn A. Walker. 1998. Centering, anaphora resolution, and discourse structure. In (Walker et al., 1998). 